
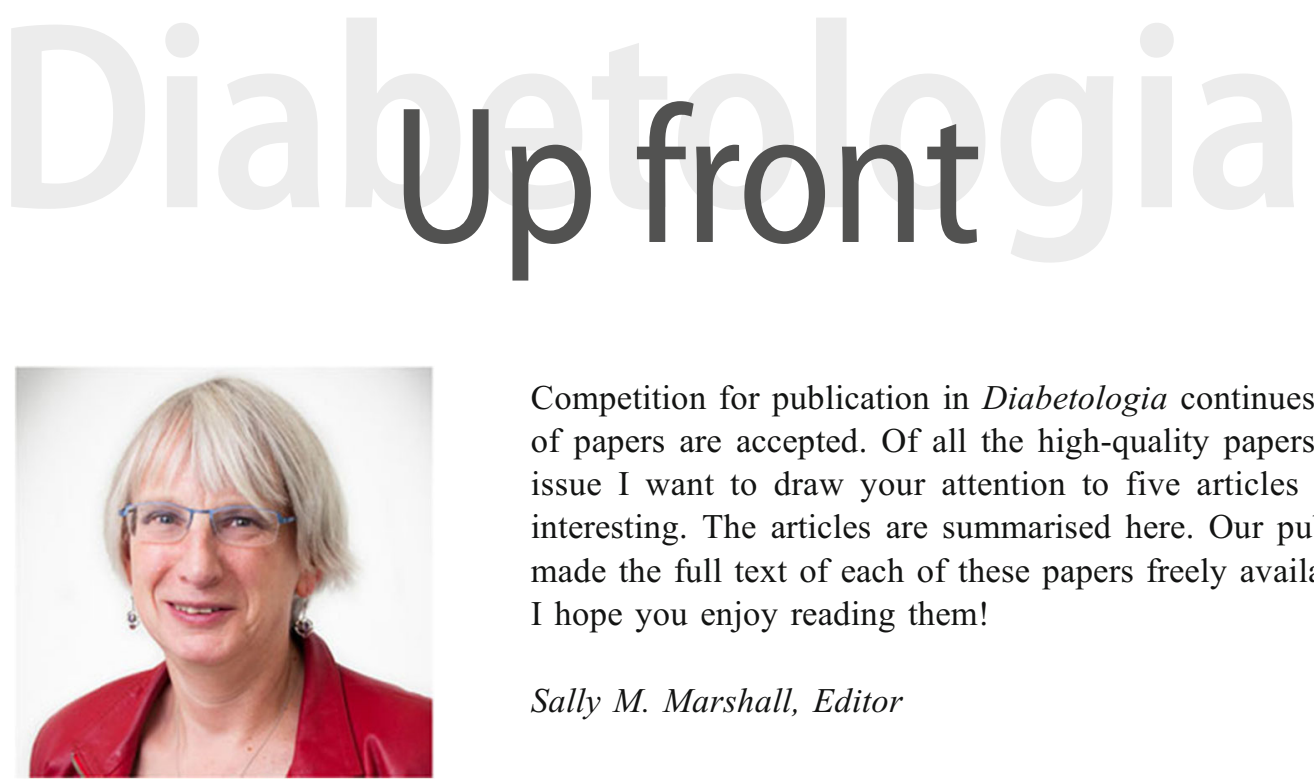

Competition for publication in Diabetologia continues to grow, and less than $20 \%$ of papers are accepted. Of all the high-quality papers that appear in this month's issue I want to draw your attention to five articles that I think are particularly interesting. The articles are summarised here. Our publisher, Springer, has kindly made the full text of each of these papers freely available.

I hope you enjoy reading them!

Sally M. Marshall, Editor

\section{Novel approaches to restore beta cell function in prediabetes and type 2 diabetes}

Vishal A. Salunkhe, Rajakrishnan Veluthakal, Steven E. Kahn, Debbie C. Thurmond

Beta cell dysfunction and/or demise are the critical components responsible for the development of prediabetes (defined as impaired fasting glucose and/or impaired glucose tolerance) and progression to frank type 2 diabetes. While tangible progress on improving beta cell function has been made, current clinical approaches do not reliably provide durable glucose control. In this issue, Salunkhe, Veluthakal and colleagues (https://doi.org/ 10.1007/s00125-018-4658-3) summarise recent advances towards improving beta cell function by improving peripheral insulin sensitivity (as a means of reducing beta cell workload). They explain how a group of factors, which in preclinical studies have been shown to multitask in both beta cells and peripheral insulin-sensitive cells, help to coordinate glucose control. The authors state that new multi-tissue-based therapeutic approaches should dovetail with efforts to formulate precision-medicine-based therapies for the variety of type 2 diabetes phenotypes. They propose that advances in genomic, epigenetic and exosome regulation of the central and tissuespecific landscape of metabolic control should facilitate efforts to refine the phenotypic cluster stratifications for optimal treatment strategies. Integration of these recent advances carries immense potential for the development of more effective medications to achieve durable glucose control in individuals with prediabetes and type 2 diabetes.

(1) The figures from this review are available as a downloadable slideset.

Neurodegeneration in diabetic retinopathy: does it really matter?

Rafael Simó, Alan W. Stitt, Thomas W. Gardner

Retinal neurodegeneration is an early event in the pathogenesis of diabetic retinopathy. In fact, the ADA has recently defined diabetic retinopathy as a highly tissue-specific neurovascular complication. In this issue, Simó et al (https://doi.org/10.1007/ s00125-018-4692-1) provide a critical review on the role of neurodegeneration in the pathogenesis of diabetic retinopathy. A special emphasis is placed on the pathophysiology of the neurovascular unit (NVU). In addition, the authors provide an overview of the usefulness of retinal assessment as an indirect method to explore brain neurodegeneration. Simó and colleagues emphasise that retinal neurodegeneration is a critical endpoint in the development of diabetic retinopathy and that neuroprotection, itself, can be considered a therapeutic target, independent of its potential impact on microvascular disease. The authors conclude that more interventional studies targeting pathogenic pathways that impact on the NVU and which offer both vaso- and neuroprotection are needed. This will be crucial for implementing a 
timely and efficient personalised medicine approach for diabetic retinopathy.

(D) The figures from this review are available as a downloadable slideset.

Efficacy and safety of methionine aminopeptidase 2 inhibition in type 2 diabetes: a randomised, placebocontrolled clinical trial

Joseph Proietto, Jaret Malloy, Dongliang Zhuang, Mark Arya, Neale D. Cohen, Ferdinandus J. de Looze, Christopher Gilfillan, Paul Griffin, Stephen Hall, Thomas Nathow, Geoffrey S. Oldfield, David N. O'Neal, Adam Roberts, Bronwyn G. A. Stuckey, Dennis Yue, Kristin Taylor, Dennis Kim

Animal and human studies indicate a beneficial effect of methionine aminopeptidase 2 (MetAP2) inhibitors on glycaemic control and other metabolic markers. In this issue, Proietto et al (https://doi.org/10.1007/s00125-018-4677-0) report results from the first study of the effects of the MetAP2 inhibitor beloranib in individuals with type 2 diabetes and obesity. The clinical trial was stopped early due to an unexpected imbalance in venous thromboembolism events in beloranib-treated vs placebo-treated individuals across beloranib clinical trials, during late-stage development of the drug. However, in individuals who had completed 26 weeks of treatment, beloranib produced statistically significant placebo-corrected reductions in both $\mathrm{HbA}_{1 \mathrm{c}}(-15.3 \mathrm{mmol} / \mathrm{mol}[-1.4 \%])$ and body weight $(-10 \%)$. The authors conclude that these data exemplify MetAP2 inhibition as a novel treatment for metabolic disease. Since this trial, a next-generation MetAP2 inhibitor with an improved safety profile has been developed and has shown encouraging efficacy and safety in an ongoing Phase 2 clinical trial in individuals with type 2 diabetes and obesity.

Association between diabetes distress and all-cause mortality in Japanese individuals with type 2 diabetes: a prospective cohort study (Diabetes Distress and Care Registry in Tenri [DDCRT 18])

Yasuaki Hayashino, Shintaro Okamura, Satoru Tsujii, Hitoshi Ishii, for the Diabetes Distress and Care Registry at Tenri Study Group

All text supplied by the authors.
Many people with diabetes feel burdened by the never-ending challenge of self-management and experience periods of frustration, anger, fear and helplessness; this is collectively referred to as diabetes distress. High levels of diabetes distress have been associated with poor glycaemic control and a high prevalence of complications in cross-sectional studies. However, there has been a lack of data on the direct association between diabetes-specific distress and all-cause mortality in individuals with diabetes. In this issue, Hayashino et al (https://doi.org/10.1007/s00125-018-4657-4) report on the association between diabetes distress and subsequent risk of all-cause mortality in a Japanese cohort of 1280 women and 2025 men with type 2 diabetes. They found that higher levels of diabetes distress were associated with subsequent risk of all-cause mortality in men with type 2 diabetes, but not in women. These findings provide new evidence to support the targeting of diabetes distress in clinical diabetes care.

\section{Magnesium deficiency prevents high-fat-diet-induced obe- sity in mice}

Steef Kurstjens, Janna A. van Diepen, Caro Overmars-Bos, Wynand Alkema, René J. M. Bindels, Frances M. Ashcroft, Cees J. J. Tack, Joost G. J. Hoenderop, Jeroen H. F. de Baaij

$\mathrm{Mg}^{2+}$ deficiency is common in type 2 diabetes, affecting approximately $30 \%$ of all individuals with this disease. Nevertheless, the metabolic consequences of hypomagnesaemia (blood $\mathrm{Mg}^{2+}<0.7 \mathrm{mmol} / \mathrm{l}$ ) remain largely unknown. In this issue, Kurstjens et al (https://doi.org/10.1007/s00125018-4680-5) demonstrate that $\mathrm{Mg}^{2+}$ deficiency in mice protects against high-fat-diet (HFD)-induced obesity, accompanied by improved insulin sensitivity and dyslipidaemia. Compared with HFD-fed mice with normal $\mathrm{Mg}^{2+}$ levels, body weight was lower in HFD-fed mice with low $\mathrm{Mg}^{2+}$ levels. This reduction in weight occurred as a result of increased lipolysis in white adipose tissue and enhanced brown adipose tissue activity. The authors propose that these effects are due to activation of the $\beta$-adrenergic system. The data demonstrate the pivotal role of $\mathrm{Mg}^{2+}$ in lipid metabolism and highlight that individuals with type 2 diabetes and hypomagnesaemia may be at particular risk for dyslipidaemia. 